\title{
NWDAF habilitando Inteligência Artificial em operações de busca e salvamento assistidas por VANTs
}

\author{
Ciro J. A. Macedo ${ }^{1,2}$, Hudson P. Romualdo ${ }^{1,4}$, Cristiano B. Both ${ }^{3}$, \\ Antonio Oliveira-Jr ${ }^{1,5}$, Kleber V. Cardoso ${ }^{1}$ \\ ${ }^{1}$ Instituto de Informática - Universidade Federal de Goiás (UFG), GO, Brasil \\ ${ }^{2}$ Instituto Federal de Goiás (IFG), GO, Brasil \\ ${ }^{3}$ Universidade Vale dos Sinos, São Leopoldo, RS, Brasil \\ ${ }^{4}$ Instituto Federal Goiano (IFGoiano), GO, Brasil \\ ${ }^{5}$ Fraunhofer Portugal AICOS, Porto, Portugal \\ ciro.macedoeifg.edu.br, hudson.romualdo@ifgoiano.edu.br \\ cbboth@unisinos.br, \{antoniojr,kleber\}@ufg.br
}

\begin{abstract}
Unmanned Aerial Vehicles (UAVs) are increasingly used for different purposes. Recent advances have made it possible to explore new applications. This article explores a context of using UAVs as part of the communication infrastructure, through the use of Artificial intelligence (AI), under an infrastructure of 6th Generation Networks. In addition, this article introduces a Virtualized Network Function (VNF) Network Data Analytics Function (NWDAF) as enabling element for Inteligência Artificial (IA) in communication networks

Resumo. Veículos Aéreos não Tripulados (VANTs) são cada vez mais utilizados para diferentes propósitos. Os recentes avanços tem possibilitado a exploração de novas aplicações. Este artigo explora um contexto de utilização de VANTs como parte da infraestrutura de comunicação, através da utilização de IA, sob uma infraestrutura de redes de $6^{\circ}$ Geração $(6 G)$. Além disso, esse artigo introduz a função de rede virtualizada NWDAF como elemento habilitador para IA em redes de comunicação.
\end{abstract}

\section{Introdução}

VANTs vem sendo cada vez mais utilizados em diferentes áreas, desempenhando as mais diversas atividades [Fotouhi et al. 2019]. Aspectos como o baixo custo de fabricação, trajetórias de voo com alta precisão e aumento da capacidade de transporte de carga útil, tem feito destes dispositivos uma ferramenta para uso em potencial em aplicações civis e militares, especialmente em operações de busca e salvamento. Tais operações são consideradas especiais e do tipo missão crítica, pois envolvem o resgate de pessoas em situação de perigo [Roldan and Ordoñez 2019]. Essas operações podem ocorrer em áreas remotas, por tempo indeterminado e, portanto, podem alcançar um alto custo, pois envolvem a mobilização de recursos especializados.

Com o objetivo de reduzir o tempo e custo dessas operações, atualmente considera-se a utilização de VANTs, atuando como parceiros na busca e salvamento, integrados com a infraestrutura de comunicação [Alsaeedy and Chong 2020]. 
Esses dispositivos podem trabalhar de forma colaborativa, estabelecendo relações simbióticas [D'Aria et al. 2021], executando um procedimento para a identificação de indivíduos através de uma técnica de visão computacional, ou explorando as bandas do espectro eletromagnético.

Para que estes dispositivos possam atuar de maneira colaborativa, torna-se fundamental atender a requisitos relacionados a posicionamento, detecção e mapeamento do ambiente. Os Key Performance Indicators (KPIs) envolvidos, estendem as funcionalidades existentes nas redes $5^{\circ}$ Geração $(5 \mathrm{G})$ em direção às redes $6 \mathrm{G}$, onde a utilização de elementos de IA, de forma distribuída e sob demanda, torna-se fundamental. Este trabalho explora o contexto das operações de busca e salvamento assistidas por VANTs, considerando a infraestrutura redes Beyond $5 G(\mathrm{~B} 5 \mathrm{G}) / 6 \mathrm{G}$ para utilizar elementos de IA como habilitadores. Em um cenário onde não existe infraestrutura de comunicação, investigase a hipótese de implantar todos os elementos do sistema de comunicação nos VANTs, utilizando Machine Learning (ML) como ferramenta de orquestração e monitoramento, conforme ilustrado na Figura 1.

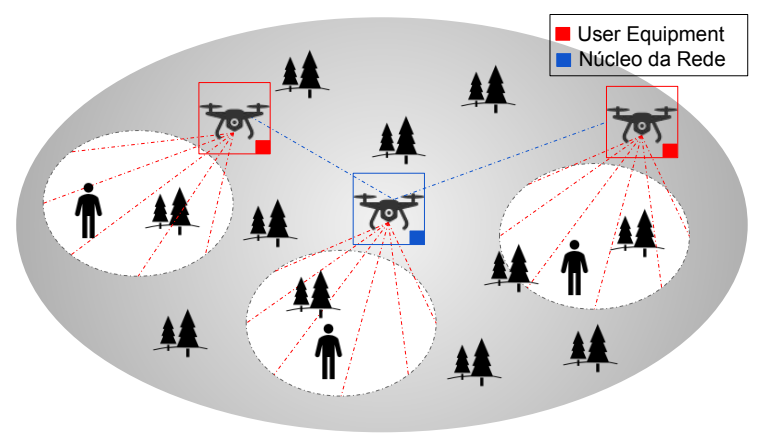

Figura 1. VANTs em busca e salvamento com os elementos do sistema 6G.

Neste contexto, esse artigo apresenta a função NWDAF como elemento habilitador para IA no âmbito das redes de comunicação B5G/ 6G. Através dessa função virtual de rede (VNF) é possível centralizar e processar as informações de todos os VANTs, realizando análises para a tomada de decisão, promovendo assim a confiabilidade necessária para a execução dessa tarefa de salvamento, que é complexa e envolve vidas.

A utilização dessa VNF ocorre em função de sua especificação. A função de análise de dados de rede (NWDAF) foi especificada como um componente funcional independente e centralizador de dados dentro do núcleo 5G. Considerando-se os dados como sendo o insumo essencial para implementação de IA, essa foi uma escolha natural.

Para além dessa Introdução, a Seção 2 apresenta um conjunto de trabalhos relacionados a área de IA, operações de busca e salvamento e redes B5G/6G. Na Seção 3, detalha um modelo habilitador voltado para a utilização de VANTs em operações de busca e salvamento, explorando a interoperabilidade das VNFs e elementos de IA. A conclusão e trabalhos futuros são discutidos na Seção 4.

\section{Trabalhos relacionados}

Diversos trabalhos discutem os desafios envolvidos, quando considera-se a utilização de VANTs como parte da infraestrutura de redes de comunicação. Os trabalhos exploram 
assuntos relacionados à computação de borda, tecnologias de comunicação e aplicação de IA como elemento habilitador [Moradi et al. 2019] [Košmerl and Vilhar 2014]. As pesquisas destacam como um dos principais benefícios, a possibilidade de um processo de implantação ágil e flexível de infraestruturas de comunicação, em regiões onde não existe cobertura. Boa parte dos trabalhos consideram VANTs, conectados a um núcleo funcional e posicionado em alguma infraestrutura física fixa no solo. Essa abordagem, embora eficiente para fins de provimento de uma infraestrutura de comunicação, é inadequada para situações de busca e salvamento por limitar o perímetro de voo dos VANTs em função destes sempre se manterem a uma certa distancia da infraestrutura de solo. Uma solução potencial seria aproveitar o conceito introduzido com a implantação de 5G que introduziu a softwarização da rede através de funções de rede virtuais (VNFs) cuja flexibilidade [Cardoso et al. 2020], permite fazer uso de um VANT para atuar como núcleo de rede. Essa abordagem é parcialmente explorada em [Fattore et al. 2020], onde os autores desacoplam a User Plane Function (UPF), posicionando-a em um VANT e, desta forma, ampliando a área de cobertura.

Neste trabalho, considera-se um grupo de VANTs, onde um deles executa não apenas uma VNF, mas sim todo o núcleo da rede. Os demais VANTs desempenham papel de User Equipments (UEs), e estão devidamente conectados ao núcleo. Com essa abordagem é possível prover uma estrutura com conectividade temporária [D’Aria et al. 2021], gerando assim uma extensão da rede principal. Trata-se de uma um rede de comunicação fisicamente móvel, onde núcleo e demais elementos precisam ser devidamente orquestrados para explorar determinada área sem perda de comunicação entre VANTs. A realização deste cenário está diretamente associada à necessidade de elementos de IA executando atividades de monitoramento e controle, considerando a dinâmica dos procedimentos envolvidos em operações de busca e salvamento.

Um problema recorrente às técnicas de IA em telecomunicações está associado ao acesso à informação. Por exemplo, diversos elementos que compõe o sistema produzem dados que desempenham papel fundamental no processo de treinamento de modelos de IA. Algumas inciativas de padronização do acesso à informação no âmbito de telecomunicações vem sendo apresentadas por 3rd Generation Partnership Project (3GPP), International Telecommunication Union (ITU) e Experiential Networked Intelligence (ENI). Dentre elas, o 3GPP em seu lançamento 17 [3GPP-TR22.829 9 09], especifica a NWDAF. Trata-se de uma VNF que tem a responsabilidade de realizar a análise dos dados produzidos pelos diversos elementos que compõem os sistemas de comunicação B5G/6G. De acordo com o especificado [Institute 2020], a NWDAF expõe um conjunto de serviços através da Service-based architecture (SBA), provendo informações estatísticas ou de predição acerca do comportamento da rede como um todo. Estas informações podem ser utilizadas como mecanismo habilitador de elementos de IA, conforme demonstrado em [Sevgican et al. 2020]. Nesse trabalho, os autores exploram as funcionalidades descritas na especificação NWDAF em um contexto de redes celulares 5G. Através da produção de um conjunto de dados sintéticos, três diferentes modelos de ML são utilizados com o objetivo de explorar o comportamento do padrão de comunicação de diferentes tipos de UEs, bem como estimativas sobre a previsão da carga de trabalho na rede $5 \mathrm{G}$.

Considerando as operações de busca e salvamento assistidas por VANTs, os KPIs 
envolvidos ultrapassam os limites do 5G. Elementos de IA são necessários como mecanismo habilitador e as funcionalidades descritas na especificação NWDAF podem estruturar um modelo habilitador minimamente funcional. Na próxima seção, apresentaremos os elementos de um modelo habilitador, destacando o papel da NWDAF, coletando e processando dados de diferentes fontes, com o objetivo de produzir informações a serem utilizadas no processo de tomada de decisão.

\section{NWDAF como habilitador para Inteligência Artificial}

Considerando o cenário ilustrado na Figura 1, o conjuntos de trabalhos recentemente publicados, bem como, as diretrizes normativas providas por 3GPP, ITU e ENI apresentados na Seção 2, argumenta-se que um modelo habilitador minimamente funcional deve considerar alguns elementos em sua composição: (i) VANTs atuando como UEs; (ii) Um VANT atuando como núcleo da rede, responsável por coordenar os demais; (iii) Dados produzidos pelos UEs durante a operação de busca; (iv) Sinalizações produzidas pelas VNFs que compõem o núcleo; (v) Interoperabilidade dos elementos que compõe os sistemas B5G/6G, em especial da arquitetura SBA; (vi) Modelos de IA/ML; (vii) Serviços expostos pela NWDAF.

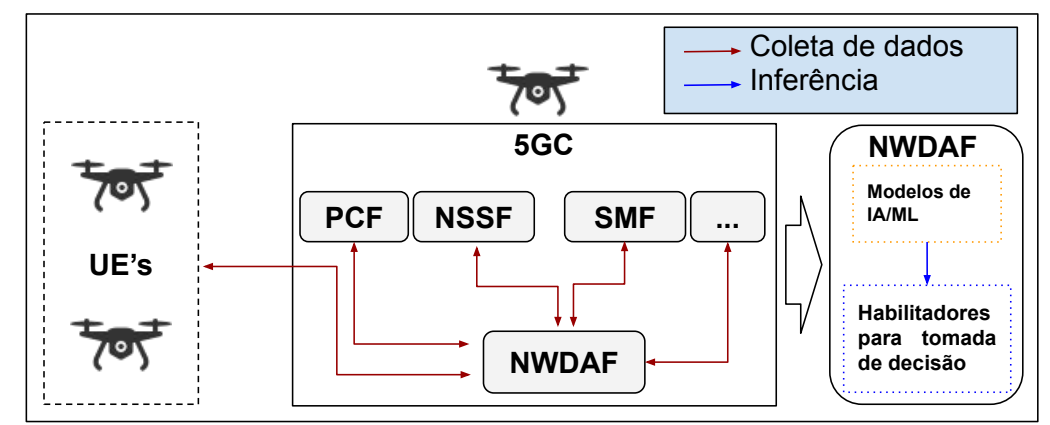

Figura 2. NWDAF como elemento habilitador de IA para VANTs.

A Figura 2 apresenta a NWDAF como habilitador de VANTs em operações de busca e salvamento. Os dados produzidos por VANTs que atuam como UEs, são transmitidos para um VANT que desempenha o papel de núcleo da rede. Neste núcleo, estão posicionadas a NWDAF e as demais VNFs. A NWDAF está conectada às demais VNFs, através da arquitetura SBA, realizando constantemente o intercâmbio de informações. As demais VNFs, alimentam os modelos de ML contidos na NWDAF com dados de sinalização. A NWDAF por sua vez, processa estes dados, produzindo informações estatísticas ou de predição que são disponibilizadas através dos serviços expostos na SBA. Estes serviços podem ser consumidas pelas demais VNFs que compõem o núcleo, e as informações podem ser utilizadas em atividades de monitoramento e controle.

A NWDAF faz parte da arquitetura especificada no lançamento 15 do 3GPP [3GPP-TR21.915 8 12]. Ela utiliza os mecanismos e interfaces especificados no núcleo $5 \mathrm{G}$, bem como, os serviços providos pelo operador do sistema com diferentes propósitos. Ela realiza a coleta de dados produzidos pelas demais VNFs durante o processo de comunicação, coleta dados contidos em repositórios, tais como Unified Data Repository (UDR), e fornece sob-demanda, informações estatísticas e de predição acerca do comportamento da rede e dos elementos que a integram. 
Diferentes algoritmos de ML podem estar contidos na NWDAF, processando os dados produzidos pelos diversos elementos do sistema de comunicação. Estes algoritmos se tornaram bastante populares e são vistos como uma solução potencial para o processamento de grandes quantidades de dados. Os modelos de ML são classificados de acordo com o método utilizado no processo de aprendizagem. São divididos em três categorias: modelos de aprendizagem supervisionada, modelos de aprendizagem não supervisionada e modelos de aprendizagem por reforço.

Dadas as características dos dados produzidos pelo processo de sinalização dos elementos que compõem o sistema B5G/6G, pode-se considerar a utilização de modelos de ML de aprendizagem supervisionada. Esta escolha se justifica pelo fato de que as especificações ENI, ITU e 3GPP descrevem de forma detalhada cada etapa do processo de sinalização. Uma implementação que obedeça estes padrões pode, portanto, ter sua estrutura de dados explorada de uma forma orientada pelas respectivas especificações, possibilitando a construção de um conjunto de dados rotulados para o treinamento de modelos supervisionados.

Considerando uma operação de busca e salvamento, as informações produzidas pela NWDAF podem ser utilizadas como elemento auxiliar no processo de tomada de decisões. Desta forma, pode-se obter informações de Quality of Service (QoS) específica, associada a uma aplicação que está sendo utilizada nos procedimentos de busca. Além disso, pode-se obter análises estatísticas ou de predição associadas ao desempenho geral da rede, obtendo informações de aspectos associados à mobilidade dos VANTs que desempenham o papel de UEs, ou ainda, obter informações da análise do padrão comportamental de comunicação entre os VANTs que desempenham o papel de UEs e o núcleo da rede. Neste último caso, com o objetivo de detectar possíveis anomalias no processo de comunicação durante a operação de busca.

\section{Considerações finais}

Este trabalho apresentou uma nova abordagem para a utilização de VANTs em operações de busca e salvamento, tendo a NWDAF como elemento habilitador. Com base nas recentes pesquisas e nas especificações produzidas pelas principais entidades de regulamentação, introduziu-se os elementos que compõem os sistemas de comunicação B5G/6G, destacando a importância da utilização de elementos de IA, em especial, dando destaque à NWDAF.

Considerando os KPIs envolvidos em um cenário B5G/6G e a disseminação do uso de elementos de IA cada vez mais distribuídos ponta-a-ponta, entre os diversos elementos que compõem o sistema de comunicação, fica evidente a necessidade de se compreender cada vez mais a correlação entre as diferentes fontes de dados existentes nos elementos que compõem o sistema B5G/6G. A NWDAF é um elemento inovador e disruptivo em redes de telecomunicações, existindo muitas questões em aberto. Com o avanço das pesquisas e consequente exploração das funcionalidades por parte da comunicada acadêmica em diferentes aplicações, atualizações na especificação técnica que descreve a estrutura e os elementos internos da NWDAF serão necessárias. Em especial, no que diz respeito à exposição de serviços adicionais na arquitetura SBA. 


\section{Agradecimentos}

O presente trabalho foi realizado com apoio do Projeto Brasil 6G, financiado pelo Ministério da Ciência, Tecnologia e Inovações (MCTI) e Rede Nacional de Pesquisa (RNP).

\section{Referências}

3GPP-TR21.915 (2018-12). Technical Specification Group Services and System Aspects; Release 15 Description. Technical report, 3rd Generation Partnership Project (3GPP). Version 0.5.0.

3GPP-TR22.829 (2019-09). Technical Specification Group Services and System Aspects; Enhancement for Unmanned Aerial Vehicles; Technical Report Stage 1 (Release 17). Technical report, 3rd Generation Partnership Project (3GPP).

Alsaeedy, A. A. R. and Chong, E. K. P. (2020). 5G and UAVs for Mission-Critical Communications: Swift Network Recovery for Search-and-Rescue Operations. Mobile Networks and Applications, 25(5):2063-2081.

Cardoso, K. V. et al. (2020). A softwarized perspective of the 5G networks.

D'Aria, G. et al. (2021). Expanded 6G vision, use cases and societal values - including aspects of sustainability, security and spectrum. Technical report, Hexa-X Project.

Fattore, U., Liebsch, M., and Bernardos, C. J. (2020). UPFlight: An enabler for Avionic MEC in a drone-extended 5G mobile network. In 2020 IEEE 91 st Vehicular Technology Conference (VTC2020-Spring), pages 1-7.

Fotouhi, A. et al. (2019). Survey on UAV Cellular Communications: Practical Aspects, Standardization Advancements, Regulation, and Security Challenges. IEEE Cоттиnications Surveys Tutorials, 21(4):3417-3442.

Institute, E. T. S. (2020). Architecture enhancements for 5G System (5GS) to support network data analytics services. Technical report, European Telecommunications Standard Institute (ETSI).

Košmerl, J. and Vilhar, A. (2014). Base stations placement optimization in wireless networks for emergency communications. In 2014 IEEE International Conference on Communications Workshops (ICC), pages 200-205.

Moradi, M. et al. (2019). Skycore: Moving Core to the Edge For Untethered and Reliable UAV-Based LTE Networks. GetMobile: Mobile Computing and Communications, 23(1):24-29.

Roldan, J. M. and Ordoñez, F. (2019). ITU Guidelines for National Emergency Telecommunication Plans; ITU-D Emergency Telecommunications - Disaster Response. Technical report, International Telecommunication Union (ITU).

Sevgican, S. et al. (2020). Intelligent network data analytics function in 5G cellular networks using machine learning. Journal of Communications and Networks, 22(3):269-280. 